\title{
Systems-level physiology of the human red blood cell is computed from metabolic and macromolecular mechanisms
}

\author{
James T. Yurkovich ${ }^{1, \dagger}$, Laurence Yang $^{2}$, and Bernhard O. Palsson ${ }^{3}$ \\ ${ }^{1}$ Institute for Systems Biology, Seattle, WA, ${ }^{2}$ Department of Chemical Engineering, Queen's University, Kingston, Canada, \\ ${ }^{3}$ Department of Bioengineering, University of California, San Diego, La Jolla, CA
}

\begin{abstract}
The human red blood cell has served as a starting point for the application and development of systems biology approaches due to its simplicity, intrinsic experimental accessibility, and importance in human health applications. Here, we present a multi-scale computational model of the human red blood cell that accounts for the full metabolic network, key proteins ( $>95 \%$ of proteome mass fraction), and several macromolecular mechanisms. Proteomics data are used to place quantitative constraints on individual protein complexes that catalyze metabolic reactions, as well as a total proteome capacity constraint. We explicitly describe molecular mechanisms-such as hemoglobin binding and the formation and detoxification of reactive oxygen species-and takes standard hematological variables (e.g., hematocrit, hemoglobin concentration) as input, allowing for personalized physiological predictions. This model is built from first principles and allows for direct computation of physiologically meaningful quantities such as the oxygen dissociation curve and an accurate computation of the flux state of the metabolic network. More broadly, this work represents an important step toward including the proteome and its function in whole-cell models of human cells.
\end{abstract}

KEYWORDS

Systems biology

Metabolism

Human red blood cell Hemoglobin binding ROS detox

\section{INTRODUCTION}

The blood is a window through which we can explore human health and disease [50]. The human red blood cell (RBC)-the most abundant human cell [46] — has historically been used as the starting point for the application and development of systems biology models due to its relative simplicity, intrinsic accessibility, and the vast amounts of data and information available on its biochemistry and physiology [52]. While the RBC lacks cellular compartments and the ability to produce energy using oxidative phosphorylation, it is heavily involved in the transport and exchange of gases throughout the body, including $\mathrm{O}_{2}, \mathrm{CO}_{2}$ [17], and NO [15]. The ability to model and compute physiological states of the RBC is thus crucial to our understanding of how our blood performs vital systems-level functions.

Since the 1970s, mathematical models have been used to study the dynamics of RBC metabolism [42]. Other modeling formalisms, like constraint-based modeling methods [51], have been used to study mechanisms underlying cellular metabolism [8, 38, 53]. These constraint-based methods have evolved to allow for the study of system dynamics [27, 48, 9], although kinetic models are best suited to exploring temporal dynamics at short time scales [20]. From the first whole-cell kinetic model of RBC metabolism in the

†james.yurkovich@isbscience.org late 1980s [22, 23, 24, 25], researchers have examined various aspects of RBC metabolism through kinetic modeling including regulatory network structure [41], the role of 2,3-diphosphoglycerate (2,3-DPG) in binding hemoglobin [32, 34, 33], hereditary glucose-6phosphate dehydrogenase (G6PDH) deficiency [35], and personalized pharmacodynamics [7]. There lies a gap, however, between what can be computationally modeled using kinetic approaches (limited by parameterization and network scale) and constraintbased approaches (limited by the completeness of the network structure).

Here, we address this gap with the development of a wholecell model of the metabolism of the human Red Blood Cell and Macromolecular Mechanisms (RBC-MM). This model is built from first principles and uses kinetic and thermodynamic constraints to represent macromolecular mechanisms such as $\mathrm{O}_{2}$ and $\mathrm{CO}_{2}$ binding to hemoglobin and the detoxification of reactive oxygen species (ROS). Further, we account for the limitation to maximum reaction velocity due to finite enzyme abundance and effective turnover rate through the integration of recently published targeted proteomic data [10]. We validate our model against previous computational models and experimental data, demonstrating the ability of the RBC-MM to compute physiologically meaningful quantities and phenotypes, such as the oxygen dissociation curve (ODC). We anticipate that the computational modeling framework presented here will help usher in a new frontier in metabolic modeling that integrates non-metabolic mechanisms to better represent 
the functions of complex biological systems.

\section{RESULTS}

We begin by detailing the construction and scope of the RBC-MM model. We then proceed to demonstrate its simulation capacity, providing validation of various computed phenotypes and quantities. Finally, we compute systems-level properties of the RBC network.

\section{Model construction}

The RBC-MM model is constructed starting from the iAB-RBC-283 metabolic network reconstruction of RBC metabolism. iAB-RBC283 represents a knowledgebase which includes 283 metabolic genes, 292 reactions, and 267 metabolites [6]. These metabolites include small molecules, cofactors, and trace minerals. For a model having $n$ reactions and $m$ metabolites, flux balance analysis (FBA) [39] computes the optimal metabolic state of a cell. A metabolic state is defined by a vector of reaction fluxes $v \in \mathbb{R}^{n}$ (in $\mathrm{mmol} / \mathrm{gDW} / \mathrm{h})$, by solving the linear program:

$$
\underset{v}{\operatorname{maximize}} c^{T} v \text { subject to } S v=0, l \leq v \leq u,
$$

where $c \in \mathbb{R}^{n}$ is the vector of objective coefficients, $S \in \mathbb{R}^{m \times n}$ is the matrix of reaction stoichiometries, and $l \in \mathbb{R}^{n}, u \in \mathbb{R}^{n}$ are the lower and upper bounds on the fluxes.

We have expanded this model to account for macromolecules present in the RBC (Figure 1). Specifically, we (i) account for the limitation to maximum reaction rate due to finite enzyme abundance and effective turnover rate and (ii) limited total protein mass in a cell. We implement these constraints in a manner similar to previous methods $[3,2]$. The FBA problem associated with this expanded model is called protein-constrained FBA ( $\mathrm{P}_{\text {con FBA }}$ [36].

In addition to fluxes, $\mathrm{P}_{\text {con }} \mathrm{FBA}$ also computes enzyme complex concentrations $e \in \mathbb{R}^{r}$ (in mmol/gDW), and protein concentrations $p \in \mathbb{R}^{s}$ (in mmol/gDW). We constrain the total protein mass $(P)$ using the molecular weight of each protein $(w)$. Each protein concentration is constrained by the measured concentration $(\Phi)$, while accounting for measurement error $(\epsilon)$. Protein concentrations are also constrained by enzyme complex stoichiometry $(a)$. Given the above constraints, the $\mathrm{P}_{\text {con }}$ FBA problem is formulated as follows:

$$
\begin{array}{ll}
\underset{e, p, v}{\operatorname{maximize}} & c^{\mathrm{T}} v \\
\text { subject to } & \mathbf{S} \cdot \mathbf{v}=0, \\
& \sum_{k} w_{k} p_{k} \leq P, \\
& p_{k} \leq \Phi_{k}^{\text {meas }}+\epsilon, \quad \forall k, \\
& p_{k} \geq \Phi_{k}^{\text {meas }}-\epsilon, \quad \forall k, \\
& v_{j} \leq \sum_{l \in \operatorname{CPLX}(j)} e_{l} k_{j, l}^{\mathrm{eff}}, \\
& p_{k}=\sum_{l \in \operatorname{CPLX}\left(p_{k}\right)} a_{k, l} e_{l}, \\
& p_{k}, v_{j} \geq 0, \quad \forall j, k,
\end{array}
$$

where $\operatorname{CPLX}(j)$ is the index set of reactions that are catalyzed by enzyme complex $j$, and $k_{j, l}^{\text {eff }}$ is the effective rate constant for the reaction $l$ being catalyzed by enzyme $j$. The model includes quantitative constraints for 306 biochemical reactions and accounts for 2,653 total proteins using published proteomic data [10].

Next, we mathematically extend this protein-constrained model with key molecular mechanisms that describe RBC physiology.
Hemoglobin mechanisms. The RBC plays a vital role in the respiratory function of blood, delivering dissolved oxygen and oxygen bound to hemoglobin (oxyhemoglobin) from the lungs to the tissues [17]. In return, tissues transport $\mathrm{CO}_{2}$ back to the lung, where carbonic anhydrase converts biocarbonate into $\mathrm{CO}_{2}$ and $\mathrm{H}_{2} \mathrm{O}$. The $\mathrm{CO}_{2}$ can be transported dissolved in the blood or can be bound to hemoglobin, depending on the concentration of $\mathrm{O}_{2}$ and $\mathrm{CO}_{2}$ in the blood [29]. The Bohr Effect [44, 4] describes how the ODC shifts based on the concentrations of $\mathrm{CO}_{2}$ and protons affect the affinity of hemologlobin for $\mathrm{O}_{2}$; the Haldane Effect describes how the ODC shifts based on the concentration of $\mathrm{O}_{2}$ affects the affinity of hemoglobin for $\mathrm{CO}_{2}$ and protons. The ODC is also dependent upon the intracellular of 2,3-diphosphoglycerate (2,3-DPG), a glycolytic intermediate and competitive inhibitor of oxygen binding to $\mathrm{Hb}$ [28].

We model the kinetics of hemoglobin $(\mathrm{Hb})$ binding with oxygen, carbon dioxide, and 2,3-DPG. We use a cooperative mechanism (i.e., the affinity for oxygen increases with more bound oxygen) with allosteric inhibition by 2,3-DPG [40]. We model the binding of $\mathrm{CO}_{2}$ to $\mathrm{Hb}$ to account for the Bohr Effect. Here we use the notation $\mathbf{H b}_{i}^{*}$ to denote a relaxed (i.e., active) form $i$ of $\mathrm{Hb}$ and the notation $\mathbf{H b}^{\dagger}$ to denote a tense (i.e., inactive) form; $i$ indicates the number of oxygen species bound to $\mathrm{Hb}$. The full reaction schema (Figure $1 \mathrm{C}$ ) is detailed below:

$$
\begin{aligned}
\mathbf{H b}_{0}^{*}+\mathrm{O}_{2} & \rightleftharpoons \mathbf{H b}_{1}^{*} \\
\mathbf{H b}_{1}^{*}+\mathrm{O}_{2} & \rightleftharpoons \mathbf{H b}_{2}^{*} \\
\mathbf{H b}_{2}^{*}+\mathrm{O}_{2} & \rightleftharpoons \mathbf{H b}_{3}^{*} \\
\mathbf{H} \mathbf{b}_{3}^{*}+\mathrm{O}_{2} & \rightleftharpoons \mathbf{H b}_{4}^{*} \\
\mathbf{H b}_{0}^{*}+23 \mathrm{DPG} & \rightleftharpoons \mathbf{D H b} \mathbf{b}^{+} \\
\mathbf{H b}_{i}^{*}-\left(\mathrm{CO}_{2}\right)_{j}+\mathrm{CO}_{2} & \rightleftharpoons \mathbf{H b}_{i}^{*}-\left(\mathrm{CO}_{2}\right)_{j+1} \\
\mathbf{H b}_{i}^{*}+\mathrm{H}^{+} & \rightleftharpoons \mathbf{H b}_{i}^{*}-\mathrm{H}^{+}
\end{aligned}
$$

where $i \in\{0,1,2,3,4\}$ represents the number of bound $\mathrm{O}_{2}$ molecules and $j \in\{0,1,2,3\}$ represents the number of bound $\mathrm{CO}_{2}$ molecules. To model these $\mathrm{Hb}$ binding kinetics, we introduce additional variables for the concentrations of $\mathrm{Hb}$ (all possible bound states), $\mathrm{O}_{2}, \mathrm{CO}_{2}, 2,3-\mathrm{DPG}, \mathrm{H}_{2} \mathrm{O}, \mathrm{OH}^{-}$, and $\mathrm{H}^{+}$.

Generation and detoxification of reactive oxygen species (ROS). $\mathrm{Hb}$ in its alternative oxygen-bound forms is prone to autooxidation [30], resulting in the formation of ROS. We reconstructed and included the following ROS generation reactions that involved $\mathrm{Hb}$ [43] (Figure 1D) in the RBC-MM model:

$$
\begin{aligned}
\mathbf{H b}_{2}^{*} & \rightarrow \mathbf{H b}_{1}^{*}+\mathrm{O}_{2}^{--} \\
\mathrm{O}_{2}^{--} & \rightarrow \mathrm{H}_{2} \mathrm{O}_{2} \\
\mathbf{H b}_{1}^{*}+\mathrm{H}_{2} \mathrm{O}_{2} & \rightarrow \mathbf{H b}(\mathrm{IV})=\mathrm{O} \\
\mathbf{H b}(\mathrm{IV})=\mathrm{O}+\mathrm{H}_{2} \mathrm{O}_{2} & \rightarrow \mathbf{H b}(\mathrm{III})-\mathrm{O}_{2}^{--} \\
\mathbf{H b}(\mathrm{III})-\mathrm{O}_{2}^{--} & \rightarrow \text { Heme degradation }+\mathrm{Fe}(\mathrm{III})
\end{aligned}
$$

where the heme degradation product results in membrane damage, particularly observed in senescent RBCs [43]. Further, we explicitly model ROS detoxification through superoxide dismutase (Equation 22) and catalase (Equation 23):

$$
\begin{aligned}
2 \mathrm{H}^{+}+2 \mathrm{O}_{2}^{--} & \rightarrow \mathrm{H}_{2} \mathrm{O}_{2}+\mathrm{O}_{2} \\
2 \mathrm{H}_{2} \mathrm{O}_{2} & \rightarrow 2 \mathrm{H}_{2} \mathrm{O}+\mathrm{O}_{2}
\end{aligned}
$$

where the rate of both reactions is extremely high $[1,43]$. 


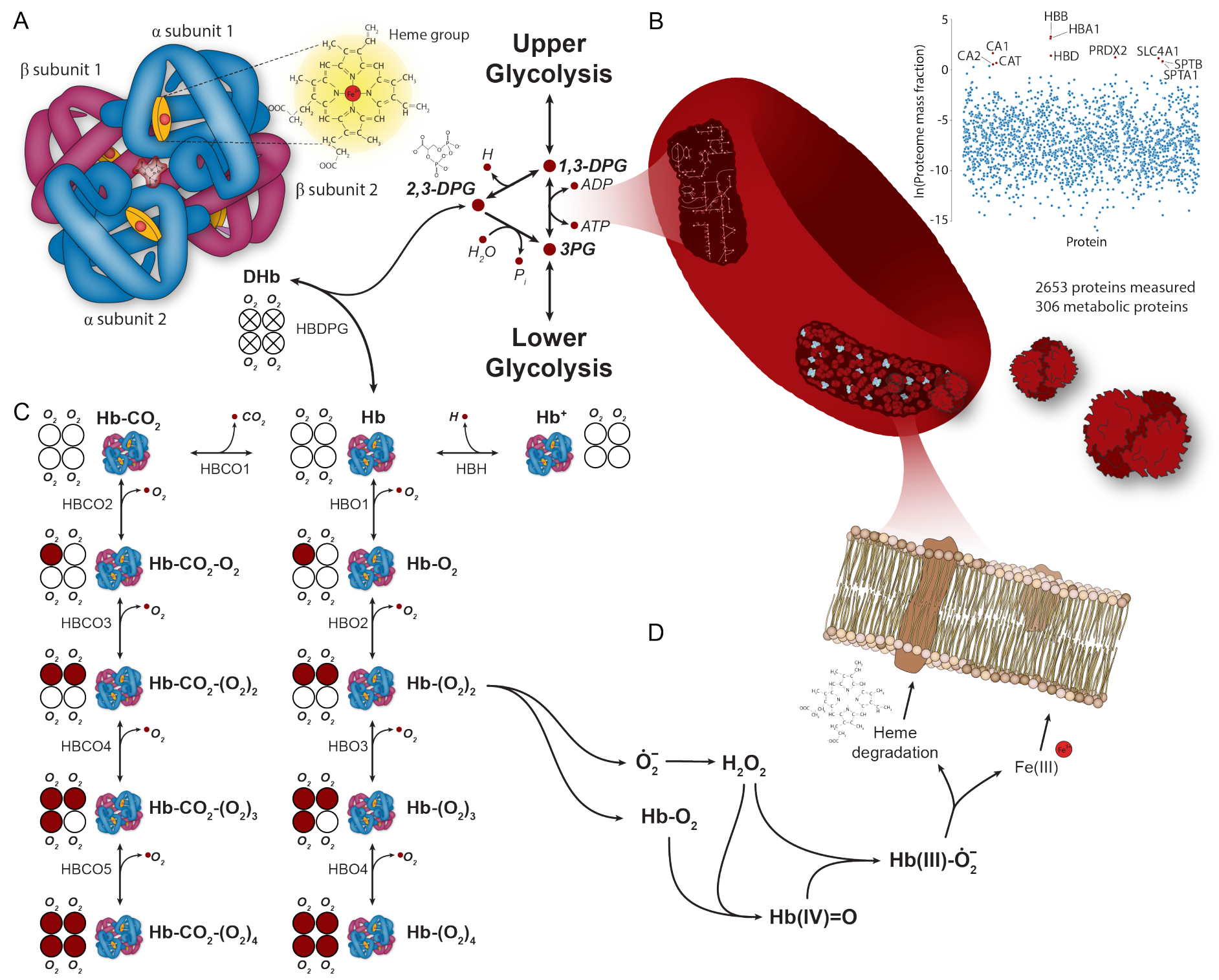

Fig. 1 Overview of the RBC-MM model's scope, including (A) integration of $\mathrm{Hb}$ reactions with the metabolic network, (B) quantitative protein constraints and crowding constraints (measured data from [10]), (C) Hb binding reactions, and (D) ROS detox mechanisms.

\section{Model simulation}

The modeling formalism described above for the RBC-MM model is built from first principles and takes a number of typically measured hematological variables as input (Table 1).

Computing the oxygen dissociation curve. We validated the physiological utility of the RBC-MM model by computing the oxygen dissociation curve (ODC) and comparing it to experimentally measured values. We explored three primary validations (Figure 2): (i) simulation of the ODC for nominal physiological values, (ii) qualitatively reproducing the Bohr Effect, and (iii) quantitatively recapitulating measured shifts due to varying concentrations of 2,3-DPG. We computed the curve using nominal baseline parameter values for the model (Table 1 ) according to the equation:

$$
\% \text { saturation }=\frac{\mathrm{O}_{2} \text { occupancy }}{\mathrm{O}_{2} \text { capacity }} \times 100
$$

where the occupancy and capacity represent all binding states of $\mathrm{Hb}$. We found that the curve is highly sensitive to the $\mathrm{K}_{\mathrm{eq}}$ values used for the binding of $\mathrm{Hb}$ to various substrates. To examine this behavior, we used values from the literature [13,19] and also fit the those parameters to measured data [47] (Figure 2A). It is not surprising that simulations using literature parameterization does not match measured data due to the differences between in vitro and in vivo $\mathrm{K}_{\text {eq }}$ values (see Supplemental Data).

Next, we tested the model's ability to qualitatively reproduce the Bohr Effect-i.e., a shift of the ODC to the left with increasing $\mathrm{pCO}_{2}$ (Figure 2B). We observed that the model did indeed reproduce this behavior, matching the same qualitative trends previously observed from Hill-type models [14, 13].

We then tested the model's ability to recapitulate the welldescribed dependence of the ODC on the intracellular concentration of 2,3-DPG [31, 26, 16]. We parameterized the model with measured values [16] using the reported hematocrit $(40 \%), \mathrm{pH}$ (7.195), and $\mathrm{Hb}$ concentration (14.75 g/dL blood); the osmotic coefficient of $\mathrm{Hb}$ [45] was used to convert the reported $\mathrm{Hb}$ concentrations. We performed a sweep across the five different concen- 

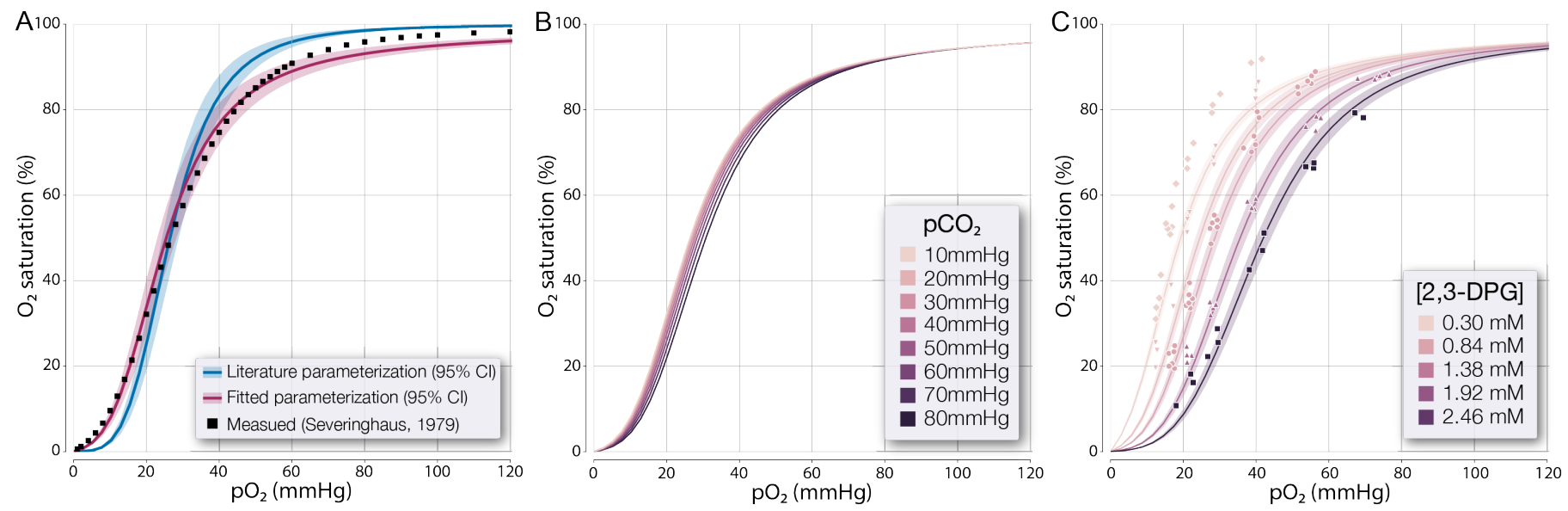

Fig. 2 Computation of the oxygen dissociation curve (ODC). (A) Baseline computation for physiological values; measured data from [47]. (B) Computing the Bohr Effect demonstrates the expected qualitative shift of the ODC to the right with increasing pCO . (C) Computing the effect of varying 2,3-DPG concentrations on the ODC; measured data from [16].

Table 1 Input parameters for the RBC-MM model; defaults are set according to literature values for hemoglobin concentration [12] and 2,3-DPG concentration [11].

\begin{tabular}{lrl}
\hline Input variable & $\begin{array}{r}\text { Default (physiological) } \\
\text { value }\end{array}$ & Units \\
\hline $\begin{array}{l}\text { Hemoglobin concen- } \\
\text { tration }\end{array}$ & 15.5 & $\mathrm{~g} / \mathrm{dL}$ blood \\
\hline Hematocrit & 45 & $\%$ \\
\hline Altitude & 0 (sea level) & $\mathrm{m}$ \\
\hline Temperature & 40 & $\mathrm{mmHg}$ \\
\hline $\begin{array}{l}\text { Partial pressure car- } \\
\text { bon dioxide }\left(\mathrm{pCO} \mathrm{C}_{2}\right)\end{array}$ & 7.24 & - \\
\hline Intracellular pH & 4.47 & $\mathrm{mmol} / \mathrm{L}$ \\
\hline $\begin{array}{l}\text { 2,3-DPG concentra- } \\
\text { tion }\end{array}$ & & \\
\hline
\end{tabular}

trations of 2,3-DPG reported by Duhm [16] (Figure 2C): 0.1, 1.9, $4.4,11.6$, and 23.0 ( $\mu$ moles $/ g$ RBC). These values were converted to units of mmol/L RBC before integration with the model. The model closely predicted the ODC for higher concentration values but underpredicted the saturation at lower concentrations. This result is likely due to parameterization of the $\mathrm{K}_{\mathrm{eq}}$ values.

Computing the concentrations of hemoglobin bound states. In the modeling formalism described here, each $\mathrm{Hb}$ form is represented as a separate variable. Thus, we can compute the concentration of each bound state of $\mathrm{Hb}$ by solving a system of linear equations based on the $\mathrm{K}_{\mathrm{eq}}$ values for each binding reaction [40]. The computed concentrations of all $\mathrm{Hb}$ bound states for the baseline simulation with fitted $\mathrm{K}_{\text {eq }}$ values (Figure $2 \mathrm{~A}$ ) are reported in Table 2. There is no experimental data with which to validate these computationally predicted values, but the values are in general agreement with estimates.

\section{DISCUSSION}

The human RBC plays a crucial role in higher-level physiological function through regulating gas exchange and transport. While a variety of modeling formalisms have been used to model some of these mechanisms, the most prevalent model type to date is a
Hill-type model [13], an empirical model. We have built a model that integrates these important macromolecular mechanisms with the complete known metabolic network of the RBC to compute functional states and phenotypic properties of the RBC. These results have several primary implications.

First, the modeling formalism presented here allows for the computation of physiological-scale phenotypes using mechanistic information. Such a framework will allow for the integration of whole-body deep-phenotyping data [50] to help identify causative factors important for the onset and progression of blood disorders. While constraint-based models have previously been used to explore whole-body physiology [5], these efforts were limited to studying the metabolic network. Here, we have extended the capabilities of the model to include non-metabolic macromolecular mechanisms that perform crucial physiological functions in our cell type of interest.

Second, we have computed the ODC of the human RBC from first principles using a model parameterized with standard hematological measurements. In other words, we model the binding of $\mathrm{Hb}$ to its various substrates using kinetic relationships instead of empirical relationships (e.g., a Hill-type model [13]). Such a modeling formalism allows for the explicit computation of the concentration of bound $\mathrm{Hb}$ forms. While the technology does not yet exist to experimentally measure these compounds, the ability to computationally predict them-while validating the overall phenotypic predictions-provides insight into the functional state of the blood's gas transport and exchange system.

Third, this framework will enable the computation of personalized phenotypic responses. The use of electronic health records for personalized medical research has been increasing over the last decade $[21,49,18]$, but we still need a unifying framework for the elucidation of causal biological mechanisms from such data [52]. The work presented here represents an important step in this direction, providing a computational network-based framework that integrates deep-phenotyping data typically recorded in a patient electronic health record (e.g., hematocrit, $\mathrm{Hb}$ concentration) to provide mechanistic predictions. By nature, these predictions offer mechanistic explanations for the observed model behavior.

Here, we have reported the most comprehensive model of the $\mathrm{RBC}$ to date that comprises the complete metabolic network, primary ROS detoxification, and $\mathrm{O}_{2}, \mathrm{CO}_{2}$, and 2,3-DPG binding to $\mathrm{Hb}$. We validated this model by computing the ODC and comparing 
bioRxiv preprint doi: https://doi.org/10.1101/797258; this version posted October 10, 2019. The copyright holder for this preprint (which was not certified by peer review) is the author/funder, who has granted bioRxiv a license to display the preprint in perpetuity. It is made available under aCC-BY-NC-ND 4.0 International license.

Table 2 Computed fractions of hemoglobin binding states (in percentages of total hemoglobin); $j \in\{0,1,2,3,4\}$ represents the number of bound $\mathrm{CO}_{2}$ molecules.

\begin{tabular}{rrrrrrrrrrrrr}
\hline $\mathbf{p O}_{2}(\mathbf{m m H g})$ & $\mathbf{H b}_{0}$ & $\mathbf{H b}_{1}$ & $\mathbf{H b}_{2}$ & $\mathbf{H b}_{3}$ & $\mathbf{H b}_{4}$ & $\mathbf{H b}-\mathrm{DPG}$ & $\mathbf{H b}_{0}-\mathbf{C O}_{2, j}$ & $\mathbf{H b}_{1}-\mathbf{C O}_{2, j}$ & $\mathbf{H b}_{2}-\mathbf{C O}_{2, j}$ & $\mathbf{H b}_{3}-\mathbf{C O}_{2, j}$ & $\mathbf{H b}_{4}-\mathbf{C O}_{2, j}$ & $\mathbf{H b}_{-} \mathbf{H}$ \\
\hline 0.00 & 0.90 & 0.00 & $1.00 \mathrm{e}-08$ & 0.00 & 0.00 & 84.52 & 8.65 & 0.00 & $1.00 \mathrm{e}-08$ & 0.00 & 0.00 & 5.93 \\
\hline 12.87 & 0.68 & 3.67 & 3.53 & 2.94 & 2.43 & 63.49 & 6.50 & 4.13 & 3.56 & 2.65 & 1.97 & 4.45 \\
\hline 25.74 & 0.29 & 3.13 & 6.04 & 10.03 & 16.60 & 27.11 & 2.77 & 3.53 & 6.08 & 9.05 & 13.47 & 1.90 \\
\hline 38.61 & 0.10 & 1.64 & 4.75 & 11.83 & 29.38 & 9.48 & 0.97 & 1.85 & 4.78 & 10.68 & 23.85 & 0.66 \\
\hline 51.48 & 0.04 & 0.86 & 3.32 & 11.02 & 36.50 & 3.73 & 0.38 & 0.97 & 3.34 & 9.95 & 29.63 & 0.26 \\
\hline 64.35 & 0.02 & 0.49 & 2.36 & 9.81 & 40.59 & 1.70 & 0.17 & 0.55 & 2.38 & 8.86 & 32.95 & 0.12 \\
\hline 77.22 & 0.01 & 0.30 & 1.74 & 8.70 & 43.20 & 0.87 & 0.09 & 0.34 & 1.75 & 7.85 & 35.07 & 0.06 \\
\hline 90.10 & 0.01 & 0.20 & 1.33 & 7.77 & 45.00 & 0.49 & 0.05 & 0.22 & 1.34 & 7.01 & 36.53 & 0.03 \\
\hline 102.97 & 0.00 & 0.14 & 1.05 & 7.00 & 46.33 & 0.30 & 0.03 & 0.15 & 1.06 & 6.32 & 37.61 & 0.02 \\
\hline 115.84 & 0.00 & 0.10 & 0.85 & 6.35 & 47.34 & 0.19 & 0.02 & 0.11 & 0.85 & 5.74 & 38.43 & 0.01 \\
\hline & & & & & & & & & & &
\end{tabular}

with experimentally measured values under different physiological conditions. The scope of this model could yet be expanded, namely through the addition of nitric oxide signaling, which will allow for additional physiologically relevant phenotypic predictions and insights. Taken together, this work represents a step toward marrying physiological deep-phenotyping data with a mechanistic computational framework capable of providing causal insights into the physiology of human blood.

\section{METHODS}

To compute the ODC, we solve the optimization problem described in Equations (1-9) as we sweep across different $\mathrm{pO}_{2}$ values. At each $\mathrm{pO}_{2}$ value, we recompute the concentrations of all $\mathrm{Hb}$ bound states. For the baseline simulations shown in Figure 2, we selected $\mathrm{K}_{\mathrm{eq}}$ values from a normal distribution using a $15 \%$ standard error 50 simulations and report the $95 \%$ confidence intervals for the 50 simulated curves, showing the mean trajectory; for the 2,3-DPG simulations, we selected $\mathrm{K}_{\mathrm{eq}}$ values from a normal distribution using a 5\% standard error for 50 simulations and report the $95 \%$ confidence intervals, showing the mean trajectory. The 2,3-DPG concentrations from [16] were converted into units of $\mathrm{mmol} / \mathrm{L}$ $\mathrm{RBC}$ using the reported density of RBC as $1.110 \mathrm{~g} / \mathrm{mL}$ [37].

Pseudo-first-order elementary rate constant (kPERC) values [40] are used to help sample the kinetically feasible flux solution space through setting constraints on reactions. We used literature values for "known" $k^{\text {cat }}$ values and sampled remaining $k^{\text {eff }}$ values from

$$
\begin{aligned}
v & =\frac{k^{\mathrm{cat}}}{K^{m}}[E][S] \\
\frac{k^{\mathrm{cat}}}{K^{m}}[S] & =\frac{v}{[E]}
\end{aligned}
$$

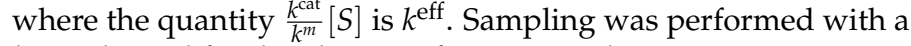
lower bound for the objective function, sodium-potassium transport $(\mathrm{NaKt})$, set to $90 \%$ of the maximum value to estimate the physiologically relevant solution space.

The concentrations of various macromolecule bound states are modeled based on $\mathrm{K}_{\mathrm{eq}}$ values where available:

$$
K_{\text {eq }}=\frac{k^{\mathrm{f}}}{k^{\mathrm{r}}}=\frac{\Pi[\text { product }]}{\Pi[\text { reactant }]} .
$$

The total mass of $\mathrm{Hb}$ is constant,

$$
\mathbf{H b}_{\text {total }}=\sum \mathrm{Hb}-\mathrm{S}
$$

where $S$ represents all bound species: $\mathrm{O}_{2}, \mathrm{CO}_{2}, 2,3-\mathrm{DPG}$, and $\mathrm{H}^{+}$. This formulation allows us to solve the system of equations represented by Equations (27) and (28) for the concentration of each bound state as previously described [40]. See the Supplemental Methods for full details on the solving procedure and model parameterization.

\section{ACKNOWLEDGEMENTS}

The authors would like to thank Zachary Haiman, Bin Du, Nathan Mih, Jared Broddrick, and Miguel Alcantar for valuable discussions. This study was funded by the Institute for Systems Biology's Translational Research Fellows Program (J.T.Y.).

[1] Abugo, O. O. and J. M. Rifkind, 1994 Oxidation of hemoglobin and the enhancement produced by nitroblue tetrazolium. J. Biol. Chem. 269: 24845-24853.

[2] Adadi, R., B. Volkmer, R. Milo, M. Heinemann, and T. Shlomi, 2012 Prediction of microbial growth rate versus biomass yield by a metabolic network with kinetic parameters. PLoS Computational Biology 8: e1002575.

[3] Beg, Q. K., A. Vazquez, J. Ernst, M. A. de Menezes, Z. Bar-Joseph, et al., 2007 Intracellular crowding defines the mode and sequence of substrate uptake by escherichia coli and constrains its metabolic activity. Proceedings of the National Academy of Sciences 104: 12663-12668.

[4] BENESCH, R. E., H. M. RANNEY, R. BENESCH, and G. M. SMITH, 1961 The chemistry of the Bohr effect. II. Some properties of hemoglobin H. J. Biol. Chem. 236: 2926-2929.

[5] Bordbar, A., A. M. Feist, R. Usaite-Black, J. Woodcock, B. O. Palsson, et al., 2011a A multi-tissue type genome-scale metabolic network for analysis of whole-body systems physiology. BMC Systems Biology 5: 180.

[6] Bordbar, A., N. Jamshidi, and B. O. Palsson, 2011b iAB-RBC-283: A proteomically derived knowledge-base of erythrocyte metabolism that can be used to simulate its physiological and patho-physiological states. BMC Syst. Biol. 5: 110.

[7] Bordbar, A., D. McCloskey, D. C. Zielinski, N. Sonnenschein, N. Jamshidi, et al., 2015 Personalized Whole-Cell kinetic models of metabolism for discovery in genomics and pharmacodynamics. Cell Syst 1: 283-292.

[8] Bordbar, A., J. M. Monk, Z. A. King, and B. O. Palsson, 2014 Constraint-based models predict metabolic and associated cellular functions. Nature Reviews Genetics 15: 107-120.

[9] Bordbar, A., J. T. Yurkovich, G. Paglia, O. Rolfsson, Ó. E. Sigurjónsson, et al. 2017 Elucidating dynamic metabolic physiology through network integration of quantitative time-course metabolomics. Sci. Rep. 7: 46249.

[10] Bryk, A. H. and J. R. Wiśniewski, 2017 Quantitative analysis of human red blood cell proteome. J. Proteome Res. 16: 2752-2761.

[11] Charache, S., S. Grisolia, A. J. Fiedler, and A. E. Hellegers, 1970 Effect of 2,3diphosphoglycerate on oxygen affinity of blood in sickle cell anemia. J. Clin. Invest. 49: 806-812.

[12] Collins, J. A., A. Rudenski, J. Gibson, L. Howard, and R. O'Driscoll, 2015 Relating oxygen partial pressure, saturation and content: the haemoglobin-oxygen dissociation curve. Breathe (Sheff) 11: 194-201.

[13] Dash, R. K. and J. B. Bassingthwaighte, 2010 Erratum to: blood hbo 2 and hbco 2 dissociation curves at varied o 2, co 2, ph, 2, 3-dpg and temperature levels. Annals of biomedical engineering 38: 1683-1701.

[14] Dash, R. K., B. Korman, and J. B. Bassingthwaighte, 2015 Simple accurate mathematical models of blood $\mathrm{HbO} 2$ and $\mathrm{HbCO} 2$ dissociation curves at varied physiological conditions: evaluation and comparison with other models. European Journal of Applied Physiology 116: 97-113.

[15] Doctor, A. and J. S. Stamler, 2011 Nitric oxide transport in blood: a third gas in the respiratory cycle. Comprehensive Physiology 1: 541-568. 
bioRxiv preprint doi: https://doi.org/10.1101/797258; this version posted October 10, 2019. The copyright holder for this preprint (which was not certified by peer review) is the author/funder, who has granted bioRxiv a license to display the preprint in perpetuity. It is made available under aCC-BY-NC-ND 4.0 International license.

[16] Duhm, J., 1971 Effects of 2, 3-diphosphoglycerate and other organic phosphate compounds on oxygen affinity and intracellular $\mathrm{pH}$ of human erythrocytes. Pflügers Archiv European Journal of Physiology 326: 341-356.

[17] Garby, L. and J. Meldon, 1977 The Respiratory Functions of Blood. Springer New York.

[18] Ginsburg, G. S. and K. A. Phillips, 2018 Precision medicine: From science to value. Health Affairs 37: 694-701.

[19] Imai, K., 1994 Adair fitting to oxygen equilibrium curves of hemoglobin. Meth Enzymol. 232: 559-576.

[20] Jamshidi, N. and B. . Palsson, 2008 Formulating genome-scale kinetic models in the post-genome era. Mol. Syst. Biol. 4: 171.

[21] Jensen, P. B., L. J. Jensen, and S. Brunak, 2012 Mining electronic health records: towards better research applications and clinical care. Nature Reviews Genetics 13: 395-405.

[22] Joshi, A. and B. O. Palsson, 1989a Metabolic dynamics in the human red cell. Part I-A comprehensive kinetic model. J. Theor. Biol. 141: 515-528.

[23] Joshi, A. and B. O. Palsson, 1989b Metabolic dynamics in the human red cell. Part II-Interactions with the environment. J. Theor. Biol. 141: 529-545.

[24] Joshi, A. and B. O. Palsson, 1990a Metabolic dynamics in the human red cell. Part III-Metabolic reaction rates. J. Theor. Biol. 142: 41-68.

[25] Joshi, A. and B. O. Palsson, 1990b Metabolic dynamics in the human red cell. Part IV-Data prediction and some model computations. J. Theor. Biol. 142: 69-85.

[26] MACDONALD, R., 1977 Red cell 2, 3-diphosphoglycerate and oxygen affinity. Anaesthesia 32: 544-553.

[27] Mahadevan, R., J. S. Edwards, and F. J. Doyle, 2002 Dynamic flux balance analysis of diauxic growth in Escherichia coli. Biophys. J. 83: 1331-1340.

[28] Meldon, J. H., 1985 pp. 63-73 in Blood Gas Transport and 2,3-DPG, edited by Kreuzer, F., S. M. Cain, Z. Turek, and T. K. Goldstick, Springer US.

[29] MELDON, J. H., P. STROEVE, and C. E. GREGOIRE, 1982 FACILITATED TRANSPORT OF CARBON DIOXIDE: A REVIEW. Chemical Engineering Communications 16: 263-300.

[30] Misra, H. P. and I. Fridovich, 1972 The generation of superoxide radical during the autoxidation of hemoglobin. J. Biol. Chem. 247: 6960-6962.

[31] MULHAUSEN, R. O., 1970 The affinity of hemoglobin for oxygen. Circulation 42: 195-198.

[32] MULQUINEY, P. J., W. A. BUBB, and P. W. KUCHEL, 1999 Model of 2, 3-bisphosphoglycerate metabolism in the human erythrocyte based on detailed enzyme kinetic equations1: in vivo kinetic characterization of 2, 3bisphosphoglycerate synthase/phosphatase using 13c and 31p NMR. Biochemical Journal 342: 567-580.

[33] MULQUINEY, P. J. and P. W. KUCHEL, 1999a Model of 2, 3-bisphosphoglycerate metabolism in the human erythrocyte based on detailed enzyme kinetic equations1: computer simulation and metabolic control analysis. Biochemical Journal 342: 597-604.

[34] MULQUINEY, P. J. and P. W. KUCHEL, 1999b Model of 2, 3-bisphosphoglycerate metabolism in the human erythrocyte based on detailed enzyme kinetic equations1: equations and parameter refinement. Biochemical Journal 342: 581-596.

[35] Nakayama, Y., A. Kinoshita, and M. Tomita, 2005 Dynamic simulation of red blood cell metabolism and its application to the analysis of a pathological condition. Theor Biol Med Model 2: 18.

[36] Nilsson, A., J. Nielsen, and B. O. Palsson, 2017 Metabolic models of protein allocation call for the kinetome. Cell Systems 5: 538-541.

[37] Norouzi, N., H. C. Bhakta, and W. H. Grover, 2017 Sorting cells by their density. PLOS ONE 12: e0180520.

[38] O'Brien, E. J., J. M. Monk, and B. O. Palsson, 2015 Using genome-scale models to predict biological capabilities. Cell 161: 971-987.

[39] Orth, J. D., I. Thiele, and B. O. Palsson, 2010 What is flux balance analysis? Nat. Biotechnol. 28: 245-248.

[40] Palsson, B. Ø., 2011 Systems Biology: Simulation of Dynamic Network States. Cambridge University Press.

[41] Price, N. D., J. L. Reed, J. A. Papin, S. J. Wiback, and B. O. Palsson, 2003 Networkbased analysis of metabolic regulation in the human red blood cell. Journal of Theoretical Biology 225: 185-194.

[42] Pujo-Menjouet, L., 2016 Blood Cell Dynamics: Half of a Century of Modelling. Mathematical Modelling of Natural Phenomena 11: 92-115.

[43] Rifkind, J. M. and E. Nagababu, 2013 Hemoglobin redox reactions and red blood cell aging. Antioxidants \& Redox Signaling 18: 2274-2283.

[44] Riggs, A. F., 1988 The bohr effect. Annual Review of Physiology 50: 181-204.

[45] Savitz, D., 1964 Osmotic properties of human red cells. The Journal of General Physiology 48: 79-94.

[46] Sender, R., S. Fuchs, and R. Milo, 2016 Revised estimates for the number of human and bacteria cells in the body. PLoS Biol. 14: e1002533.

[47] Severinghaus, J., 1979 Simple, accurate equations for human blood o2 dissociation computations. J Appl Physiol Respir Environ Exerc Physiol 46: 599-602.

[48] Waldherr, S., D. A. Oyarzún, and A. Bockmayr, 2015 Dynamic optimization of metabolic networks coupled with gene expression. Journal of theoretical biology 365: 469-485.

[49] Wu, P. Y., C. W. Cheng, C. D. Kaddi, J. Venugopalan, R. Hoffman, et al., 2017 -Omic and Electronic Health Record Big Data Analytics for Precision Medicine. IEEE Trans Biomed Eng 64: 263-273.

[50] Yurkovich, J. T. and L. Hood, 2019 Blood is a window into health and disease. Clinical Chemistry p. clinchem.2018.299065.

[51] Yurkovich, J. T. and B. O. Palsson, 2016 Solving puzzles with missing pieces: The power of systems biology. Proceedings of the IEEE 104: 2-7.

[52] Yurkovich, J. T. and B. O. Palsson, 2018 Quantitative -omic data empowers bottom-up systems biology. Current Opinion in Biotechnology 51: 130-136.

[53] Yurkovich, J. T., D. C. Zielinski, L. Yang, G. Paglia, O. Rolfsson, et al., 2017 Quantitative time-course metabolomics in human red blood cells reveal the temperature dependence of human metabolic networks. J. Biol. Chem. . 